\title{
Variable Hardy and Hardy-Lorentz spaces and applications in Fourier analysis
}

\author{
Ferenc Weisz
}

\begin{abstract}
We summarize some results about the variable Hardy and HardyLorentz spaces $H_{p(\cdot)}\left(\mathbb{R}^{d}\right)$ and $H_{p(\cdot), q}\left(\mathbb{R}^{d}\right)$ and about the $\theta$-summability of multidimensional Fourier transforms. We prove that the maximal operator of the $\theta$ means is bounded from $H_{p(\cdot)}\left(\mathbb{R}^{d}\right)$ to $L_{p(\cdot)}\left(\mathbb{R}^{d}\right)$ and from $H_{p(\cdot), q}\left(\mathbb{R}^{d}\right)$ to $L_{p(\cdot), q}\left(\mathbb{R}^{d}\right)$. This implies some norm and almost everywhere convergence results for the Riesz, Bochner-Riesz, Weierstrass, Picard and Bessel summations.
\end{abstract}

Mathematics Subject Classification (2010): 42B08, 42A38, 42A24, 42B25, 42B30.

Keywords: Variable Hardy spaces, variable Hardy-Lorentz spaces, atomic decomposition, $\theta$-summability, maximal operator.

\section{Introduction}

It was proved in Stein, Taibleson and Weiss [27] that the Bochner-Riesz means

$$
\sigma_{T}^{\alpha} f(x):=\int_{-T}^{T}\left(1-\left(\frac{|t|}{T}\right)^{2}\right)^{\alpha} \widehat{f}(t) e^{2 \pi \imath x t} d t \quad(x \in \mathbb{R}, T>0)
$$

converge almost everywhere to $f$, whenever the one-dimensional function $f \in L_{p}\left(\mathbb{R}^{d}\right)$ $(1 \leq p<\infty, 0<\alpha<\infty)$. Here $\widehat{f}$ denotes the Fourier transform of $f$. Moreover, the maximal operator $\sigma_{*}^{\theta}$ of the Bochner-Riesz means is bounded from the Hardy space $H_{p}(\mathbb{R})$ to $L_{p}(\mathbb{R})$ if $p>1 /(\alpha+1)$ (see also Grafakos [12] and Lu [20] or Weisz [33]).

In this paper, we generalize these results to multi-dimensional functions, to Lebesgue and Hardy spaces with variable exponents and to a general method of summation, to the $\theta$-summability. The $\theta$-summation is generated by a single function $\theta$ and includes all well known summations. This topic is studied intensively in the literature (see e.g. Butzer and Nessel [2], Trigub and Belinsky [29], Gát [8, 9], Goginava

This research was supported by the Hungarian Scientific Research Funds (OTKA) No K115804. This paper was presented at the 12th Joint Conference on Mathematics and Computer Science (MaCS 2018), June 14-17, 2014, Cluj-Napoca, Romania. 
[10, 11], Persson, Tephnadze and Wall [22], Simon [24, 25] and Feichtinger and Weisz $[7,31,32,33]$ and the references therein).

Let $p(\cdot): \mathbb{R}^{n} \rightarrow(0, \infty)$ be a variable exponent function satisfying the globally log-Hölder condition and $0<q \leq \infty$. We introduce the variable Lebesgue, Lorentz, Hardy and Hardy-Lorentz spaces $L_{p(\cdot)}\left(\mathbb{R}^{d}\right), L_{p(\cdot), q}\left(\mathbb{R}^{d}\right), H_{p(\cdot)}\left(\mathbb{R}^{d}\right)$ and $H_{p(\cdot), q}\left(\mathbb{R}^{d}\right)$. These spaces are investigated very intensively in the literature nowadays (see e.g. Cruz-Uribe and Fiorenza [5], Diening at al. [6], Kempka and Vybral [16], Nakai and Sawano [21, 23], Jiao at al. [14, 15], Yan at al. [35] and Liu at al. [18, 19]). We give the atomic decomposition of these Hardy spaces just mentioned. If $p(\cdot)$ is a constant, then we get back the classical Lebesgue and Hardy spaces. Under some conditions on $\theta$, we will prove that the maximal operator $\sigma_{*}^{\theta}$ is bounded from $H_{p(\cdot)}\left(\mathbb{R}^{d}\right)$ to $L_{p(\cdot)}\left(\mathbb{R}^{d}\right)$ and from $H_{p(\cdot), q}\left(\mathbb{R}^{d}\right)$ to $L_{p(\cdot), q}\left(\mathbb{R}^{d}\right)$ for all $p(\cdot)>p_{0}$. As a consequence, we obtain some norm and almost everywhere convergence results for the $\theta$-means. As special cases of the $\theta$-summation, we consider the Riesz, Bochner-Riesz, Weierstrass, Picard and Bessel summations. This paper was the base of my talk given at the 12th Joint Conference on Mathematics and Computer Science, Cluj-Napoca, June 2018.

\section{2. $\theta$-summability of Fourier transforms}

For a constant $p$, the $L_{p}\left(\mathbb{R}^{d}\right)$ space is equipped with the quasi-norm

$$
\|f\|_{L_{p}\left(\mathbb{R}^{d}\right)}:=\left(\int_{\mathbb{R}^{d}}|f(x)|^{p} d x\right)^{1 / p} \quad(0<p<\infty),
$$

with the usual modification for $p=\infty$. Here we integrate with respect to the Lebesgue measure $\lambda$. The Lebesgue measure of a set $H$ will be denoted also by $|H|$.

The Fourier transform of a function $f \in L_{1}\left(\mathbb{R}^{d}\right)$ is defined by

$$
\widehat{f}(x):=\int_{\mathbb{R}^{d}} f(t) e^{-2 \pi \imath x \cdot t} d t \quad\left(x \in \mathbb{R}^{d}\right),
$$

where $\imath=\sqrt{-1}$ and $x \cdot t:=\sum_{k=1}^{d} x_{k} t_{k}$. Suppose first that $f \in L_{p}\left(\mathbb{R}^{d}\right)$ for some $1 \leq p \leq 2$. The Fourier inversion formula

$$
f(x)=\int_{\mathbb{R}^{d}} \widehat{f}(t) e^{2 \pi \imath x \cdot t} d t \quad\left(x \in \mathbb{R}^{d}\right)
$$

holds if $\widehat{f} \in L_{1}\left(\mathbb{R}^{d}\right)$. This motivates the definition of the Dirichlet integral $s_{T} f$ defined by

$$
s_{T} f(x):=\int_{\mathbb{R}^{d}} \chi_{\{|t| \leq T\}} \widehat{f}(t) e^{2 \pi \imath x \cdot t} d t \quad\left(x \in \mathbb{R}^{d}\right) .
$$

It is known that, when $d=1$ and $1<p<\infty$, for any one-dimensional function $f \in L_{p}(\mathbb{R})$,

$$
\lim _{T \rightarrow \infty} s_{T} f=f \quad \text { in the } L_{p}(\mathbb{R}) \text {-norm and almost everywhere. }
$$

The almost everywhere convergence result in (2.2), due to Carleson [3] and Hunt [13] (see also Grafakos [12]), is one of the deepest results in harmonic analysis. It is also known that the convergence in (2.2) does not hold true for any higher dimensional 
function $f \in L_{p}\left(\mathbb{R}^{d}\right)$, except the norm convergence for $p=2$ (see Stein and Weiss [28] or Grafakos [12]). On the other hand, the convergence in (2.2) does not hold true for $p=1$ even when $d=1$. This motivates one to replace the Dirichlet integrals by some summability means, which are defined via replacing the characteristic function in (2.1) by various functions with higher regularity. Via this replacement of the Dirichlet integrals by some summability means, we will extend $(2.2)$ to the case $p \leq 1$.

Now we introduce the definition of $\theta$-summability, which is a general summation generated by a single function $\theta:[0, \infty) \rightarrow \mathbb{R}$. Let $\theta_{0}(x):=\theta(|x|)$ and suppose that

$$
\theta \in C_{0}[0, \infty), \quad \theta(0)=1, \quad \theta_{0} \in L_{1}\left(\mathbb{R}^{d}\right), \quad \widehat{\theta_{0}} \in L_{1}\left(\mathbb{R}^{d}\right),
$$

where $C_{0}[0, \infty)$ denotes the spaces of continuous functions vanishing at infinity and $|\cdot|$ denotes the Euclidean norm. For $T>0$, the $T$ th $\theta$-mean of the function $f \in L_{p}\left(\mathbb{R}^{d}\right)$ $(1 \leq p \leq 2)$ is given by

$$
\sigma_{T}^{\theta} f(x):=\int_{\mathbb{R}^{d}} \theta\left(\frac{|t|}{T}\right) \widehat{f}(t) e^{2 \pi \imath x \cdot t} d t \quad\left(x \in \mathbb{R}^{d}, T>0\right) .
$$

This integral is well defined because $\theta_{0} \in L_{p}\left(\mathbb{R}^{d}\right)$ and $\widehat{f} \in L_{p^{\prime}}\left(\mathbb{R}^{d}\right)$, where

$$
1 / p+1 / p^{\prime}=1 \text {. }
$$

For an integrable function $f$, it is known that we can rewrite $\sigma_{T}^{\theta} f$ as

$$
\sigma_{T}^{\theta} f(x)=\int_{\mathbb{R}^{d}} f(x-t) K_{T}^{\theta}(t) d t=f * K_{T}^{\theta}(x) \quad\left(x \in \mathbb{R}^{d}, T>0\right),
$$

where $*$ denotes the convolution and the $T$ th $\theta$-kernel is given by

$$
K_{T}^{\theta}(x):=\int_{\mathbb{R}^{d}} \theta\left(\frac{|t|}{T}\right) e^{2 \pi \imath x \cdot t} d t=T^{d} \widehat{\theta}_{0}(T x) \quad\left(x \in \mathbb{R}^{d}, T>0\right) .
$$

We can extend the $\theta$-means to all function spaces investigated in this paper by

$$
\sigma_{T}^{\theta} f:=f * K_{T}^{\theta} \quad(T>0)
$$

The maximal $\theta$-operator is introduced by

$$
\sigma_{*}^{\theta} f:=\sup _{T>0}\left|\sigma_{T}^{\theta} f\right|
$$

\section{One-dimensional Hardy spaces $H_{p}(\mathbb{R})$}

For a Schwartz function $\psi \in S(\mathbb{R})$ with $\int_{\mathbb{R}} \psi d \lambda \neq 0$ and a tempered distribution $f \in S^{\prime}(\mathbb{R})$ let the maximal function $f^{+}$be defined by

$$
f^{+}(x):=\sup _{0<t<\infty}\left|\left(f * \psi_{t}\right)(x)\right| \quad(x \in \mathbb{R}),
$$

where

$$
\psi_{t}(x):=t^{-1} \psi(x / t) \quad(t>0) .
$$

A tempered distribution $f \in S^{\prime}\left(\mathbb{R}^{d}\right)$ is in the Hardy spaces $H_{p}(\mathbb{R})(0<p \leq \infty)$ if

$$
\|f\|_{H_{p}(\mathbb{R})}:=\left\|f^{+}\right\|_{L_{p}(\mathbb{R})}<\infty .
$$


For different Schwartz functions $\psi$, we get the same Hardy space with equivalent norms. The following theorem is well known (see e.g. Stein [26] or Weisz [33]).

Theorem 3.1. If $1<p \leq \infty$, then the Hardy space $H_{p}(\mathbb{R})$ is equivalent to $L_{p}(\mathbb{R})$, i.e.,

$$
H_{p}(\mathbb{R}) \sim L_{p}(\mathbb{R}) .
$$

The atomic decomposition is a useful characterization of the Hardy spaces by the help of which some boundedness results, duality theorems, inequalities and interpolation results can be proved. The atomic decomposition of Hardy spaces were proved e.g. in Latter [17], Lu [20], Wilson [34], Stein [26] and Weisz [33].

Definition 3.2. Let $0<p<\infty$ and fix an integer $1 / p-1<s<\infty$. A measurable function $a$ is called a $p$-atom if there exists an interval $B \subset \mathbb{R}$ such that

(a) supp a $\subset \mathrm{B}$,

(b) $\|a\|_{L_{\infty}(\mathbb{R})} \leq|B|^{-1 / p}$,

(c) $\int_{\mathbb{R}} a(x) x^{\alpha} d x=0$ for all natural numbers $\alpha \leq s$.

Every function from the Hardy space $H_{p}(\mathbb{R})(0<p \leq 1)$ can be decomposed into the sum of atoms.

Theorem 3.3. Let $0<p \leq 1$. A tempered distribution $f \in S^{\prime}(\mathbb{R})$ is in $H_{p}(\mathbb{R})$ if and only if there exist a sequence $\left\{a_{i}\right\}_{i \in \mathbb{N}}$ of p-atoms and a sequence $\left\{\lambda_{i}\right\}_{i \in \mathbb{N}}$ of positive numbers such that

$$
f=\sum_{i \in \mathbb{N}} \lambda_{i} a_{i} \quad \text { in } \quad S^{\prime}(\mathbb{R})
$$

Moreover,

$$
\|f\|_{H_{p}(\mathbb{R})} \sim \inf \left(\sum_{i \in \mathbb{N}} \lambda_{i}^{p}\right)^{1 / p},
$$

where the infimum is taken over all decompositions of $f$ as above.

In the present form the theorem does not hold for $1<p<\infty$ and it cannot be extended to variable Hardy spaces. However, using the following ideas, we will extend the atomic decomposition to all $0<p<\infty$ and to variable Hardy spaces in Section 5. First of all observe that (ii) of Definition 3.2 is the same as

$$
\|a\|_{L_{\infty}(\mathbb{R})} \leq \frac{1}{\left\|\chi_{B}\right\|_{L_{p}(\mathbb{R})}} .
$$

Secondly, for $0<p \leq 1$, (3.1) can be written as

$$
\|f\|_{H_{p}(\mathbb{R})} \sim \inf \left(\sum_{i \in \mathbb{N}} \lambda_{i}^{p}\right)^{1 / p}=\inf \left\|\left(\sum_{i \in \mathbb{N}}\left(\frac{\lambda_{i} \chi_{B_{i}}}{\left\|\chi_{B_{i}}\right\|_{L_{p}(\mathbb{R})}}\right)^{p}\right)^{1 / p}\right\|_{L_{p}(\mathbb{R})},
$$

where $B_{i}$ is the support of the $p$-atom $a_{i}$. This form of the atomic decomposition can already be extended to variable Hardy spaces.

It is an important problem as to whether a sublinear operator $V$ is bounded from the Hardy space $H_{p}(\mathbb{R})$ to $L_{p}(\mathbb{R})$. If this boundedness holds for at least one $p$ with $p<1$ and for at least one $p$ with $p>1$, then we obtain by interpolation that $V$ 
is of weak type $(1,1)$, which is a basic inequality in harmonic analysis. The following (falls) theorem can be found several times in the literature.

Theorem 3.4. Suppose that $0<p \leq 1, V$ is a sublinear operator and

$$
\|V a\|_{L_{p}(\mathbb{R})} \leq C_{p}
$$

for all p-atoms a. Then

$$
\|V f\|_{L_{p}(\mathbb{R})} \leq C_{p}\|f\|_{H_{p}(\mathbb{R})} \quad\left(f \in H_{p}(\mathbb{R})\right) .
$$

Here, we give a typical proof of this theorem. Usually, we take an atomic decomposition

$$
f=\sum_{i \in \mathbb{N}} \lambda_{i} a_{i}
$$

where each $a_{i}$ is a $p$-atom and

$$
\left(\sum_{i \in \mathbb{N}} \lambda_{i}^{p}\right)^{1 / p} \leq C_{p}\|f\|_{H_{p}(\mathbb{R})}
$$

Then

$$
|V f| \leq \sum_{i \in \mathbb{N}} \lambda_{i}\left|V a_{i}\right|
$$

and

$$
\|V f\|_{p}^{p} \leq \sum_{i \in \mathbb{N}} \lambda_{i}^{p}\left\|V a_{i}\right\|_{p}^{p} \leq C_{p}\|f\|_{H_{p}(\mathbb{R})}^{p} \quad(0<p \leq 1) .
$$

The problem is that this proof is falls because the inequality (3.3) does not necessarily hold. Indeed, Bownik [1] have given an operator $V$ for which (3.3) and Theorem 3.4 do not hold.

Now we present one correct version of Theorem 3.4 (see Weisz [33]). In summability theory, we investigate often the operators

$$
V_{t} f(x)=f * K_{t}(x):=\int_{\mathbb{R}} f(u) K_{t}(x-u) d u \quad(t>0),
$$

where $K_{t} \in L_{1}(\mathbb{R})$ are summability kernels. Then $V_{t}: L_{1}(\mathbb{R}) \rightarrow L_{1}(\mathbb{R})$ are bounded linear operators. Set

$$
V_{*} f:=\sup _{t>0}\left|V_{t} f\right| .
$$

Let us denote by $2 I$ the interval with the same center as $I$ and the radius of $2 I$ is two times the radius of $I$.

Theorem 3.5. Let $0<p \leq 1, K_{t} \in L_{1}(\mathbb{R})$ and $V_{t} f=f * K_{t}$. Suppose that

$$
\int_{\mathbb{R} \backslash 2 B}\left|V_{*} a\right|^{p} d \lambda \leq C_{p}
$$

for all p-atoms a with support $B$. If $V_{*}$ is bounded from $L_{\infty}(\mathbb{R})$ to $L_{\infty}(\mathbb{R})$, then

$$
\|V f\|_{L_{p}(\mathbb{R})} \leq C_{p}\|f\|_{H_{p}(\mathbb{R})} \quad\left(f \in H_{p}(\mathbb{R})\right) .
$$


It is easy to see that (3.4) implies (3.2). Indeed, using (ii) of Definition 3.2 and the boundedness of $V_{*}$ on $L_{\infty}(\mathbb{R})$, we obtain

$$
\int_{\mathbb{R}}\left|V_{*} a\right|^{p} d \lambda \leq \int_{2 B}\left|V_{*} a\right|^{p} d \lambda+C_{p} \leq C_{p} .
$$

One of the most investigated summability is the Bochner-Riesz summability, defined by

$$
\theta_{0}(t)=\left\{\begin{array}{ll}
\left(1-|t|^{2}\right)^{\alpha}, & \text { if }|t|>1 ; \\
0, & \text { if }|t| \leq 1
\end{array} \quad(t \in \mathbb{R})\right.
$$

where $0<\alpha<\infty$. It was introduced already in the Introduction. The next result was proved in Stein, Taibleson and Weiss [27], Grafakos [12] and Lu [20]. [27] contains a counterexample which shows that the theorem is not true for $p \leq 1 / \alpha+1$.

Corollary 3.6. If $\frac{1}{\alpha+1}<p<\infty$, then for the Bochner-Riesz means we have

$$
\left\|\sigma_{*}^{\theta} f\right\|_{L_{p}(\mathbb{R})} \lesssim\|f\|_{H_{p}(\mathbb{R})} \quad\left(f \in H_{p}(\mathbb{R})\right) .
$$

In the next sections, we will generalize these theorems to higher dimensional functions and to Hardy spaces with variable exponents.

\section{Variable Lebesgue and Lorentz spaces}

We are going to generalize the $L_{p}(\mathbb{R})$ spaces. A measurable function $p(\cdot): \mathbb{R}^{d} \rightarrow$ $(0, \infty)$ is called a variable exponent if

$$
0<p_{-}:=\operatorname{essinf}_{x \in \mathbb{R}^{d}} p(x) \quad \text { and } \quad p_{+}:=\underset{x \in \mathbb{R}^{d}}{\operatorname{ess} \sup } p(x)<\infty .
$$

The variable Lebesgue space $L_{p(\cdot)}\left(\mathbb{R}^{d}\right)$ contains all measurable functions $f$, for which

$$
\|f\|_{L_{p(\cdot)}\left(\mathbb{R}^{d}\right)}:=\inf \left\{\rho \in(0, \infty): \int_{\mathbb{R}^{d}}\left(\frac{\mid f(x)}{\rho}\right)^{p(x)} d x \leq 1\right\}<\infty .
$$

If $p(\cdot)$ is a constant, then we get back the $L_{p}\left(\mathbb{R}^{d}\right)$ spaces. Usually, we cannot compute exactly the $L_{p(\cdot)}\left(\mathbb{R}^{d}\right)$-norm of a function or even of a characteristic function. However, we know the following inequalities due to Cruz-Uribe and Fiorenza [5, Corollary 2.23]:

$$
\left(\int_{\mathbb{R}^{d}}|f(x)|^{p(x)} d x\right)^{1 / p_{+}} \leq\|f\|_{L_{p(\cdot)}\left(\mathbb{R}^{d}\right)} \leq\left(\int_{\mathbb{R}^{d}}|f(x)|^{p(x)} d x\right)^{1 / p_{-}}
$$

if $\|f\|_{L_{p(\cdot)}\left(\mathbb{R}^{d}\right)} \geq 1$ and

$$
\left(\int_{\mathbb{R}^{d}}|f(x)|^{p(x)} d x\right)^{1 / p_{-}} \leq\|f\|_{L_{p(\cdot)}\left(\mathbb{R}^{d}\right)} \leq\left(\int_{\mathbb{R}^{d}}|f(x)|^{p(x)} d x\right)^{1 / p_{+}}
$$

if $\|f\|_{L_{p(\cdot)}\left(\mathbb{R}^{d}\right)} \leq 1$. 
We denote by $C^{\log }\left(\mathbb{R}^{d}\right)$ the set of all variable exponents $p(\cdot)$ satisfying the socalled globally log-Hölder continuous condition, namely, there exist two positive constants $C_{\log }(p)$ and $C_{\infty}$, and $p_{\infty} \in \mathbb{R}$ such that, for any $x, y \in \mathbb{R}^{d}$,

$$
|p(x)-p(y)| \leq \frac{C_{\log }(p)}{\log (e+1 /|x-y|)}
$$

and

$$
\left|p(x)-p_{\infty}\right| \leq \frac{C_{\infty}}{\log (e+|x|)} .
$$

It is easy to see that a Lipschitz function of order $\alpha(0<a \leq 1)$ satisfies (4.1).

Given a locally integrable function $f$, the Hardy-Littlewood maximal operator $M$ is defined by

$$
M f(x):=\sup _{x \in B} \frac{1}{|B|} \int_{B}|f(y)| d y \quad\left(x \in \mathbb{R}^{d}\right),
$$

where the supremum is taken over all balls $B$ of $\mathbb{R}^{d}$ containing $x$. It is known that $M$ is bounded on $L_{p}\left(\mathbb{R}^{d}\right)$ if $1<p<\infty$ and is of weak type $(1,1)$. This is extended to the variable Lebesgue spaces as follows (Cruz-Uribe and Fiorenza [5, Theorem 3.16]).

Theorem 4.1. Suppose that $p(\cdot) \in C^{\log }\left(\mathbb{R}^{d}\right)$ and $f \in L_{p(\cdot)}\left(\mathbb{R}^{d}\right)$. If $p_{-} \geq 1$, then

$$
\sup _{\rho \in(0, \infty)}\left(\rho\left\|\chi_{\left\{x \in \mathbb{R}^{d}: M f(x)>\rho\right\}}\right\|_{L_{p(\cdot)}\left(\mathbb{R}^{d}\right)}\right) \leq\|f\|_{L_{p(\cdot)}\left(\mathbb{R}^{d}\right)} .
$$

If in addition $p_{-}>1$, then

$$
\|M f\|_{L_{p(\cdot)}\left(\mathbb{R}^{d}\right)} \leq C\|f\|_{L_{p(\cdot)}\left(\mathbb{R}^{d}\right)} .
$$

We recall the Fefferman-Stein vector-valued inequality on variable Lebesgue spaces, which is a generalization of inequality (4.2) and is used in the proof of Theorem 6.1 (for the proof see Cruz-Uribe at al. [4, Corollary 2.1]).

Theorem 4.2. If $p(\cdot) \in C^{\log }\left(\mathbb{R}^{d}\right)$ with $p_{-}>1$ and $1<r<\infty$, then

$$
\left\|\left(\sum_{j=1}^{\infty}\left(M f_{j}\right)^{r}\right)^{1 / r}\right\|_{L_{p(\cdot)}\left(\mathbb{R}^{d}\right)} \leq C\left\|\left(\sum_{j=1}^{\infty}\left|f_{j}\right|^{r}\right)^{1 / r}\right\|_{L_{p(\cdot)}\left(\mathbb{R}^{d}\right)} .
$$

The variable Lorentz spaces were introduced and investigated by Kempka and Vybral [16]. $L_{p(\cdot), q}\left(\mathbb{R}^{d}\right)$ is defined to be the space of all measurable functions $f$ such that

$$
\|f\|_{L_{p(\cdot), q}\left(\mathbb{R}^{d}\right)}:= \begin{cases}\left(\int_{0}^{\infty} \rho^{q}\left\|\chi_{\left\{x \in \mathbb{R}^{d}:|f(x)|>\rho\right\}}\right\|_{L_{p(\cdot)}\left(\mathbb{R}^{d}\right)}^{q} \frac{d \rho}{\rho}\right)^{1 / q}, & \text { if } 0<q<\infty ; \\ \sup _{\rho \in(0, \infty)} \rho\left\|\chi_{\left\{x \in \mathbb{R}^{d}:|f(x)|>\rho\right\}}\right\|_{L_{p(\cdot)}\left(\mathbb{R}^{d}\right)}, & \text { if } q=\infty\end{cases}
$$

is finite. If $p(\cdot)$ is a constant, we get back the classical Lorentz spaces and $L_{p, p}\left(\mathbb{R}^{d}\right)=$ $L_{p}\left(\mathbb{R}^{d}\right)$. This last equality is not true for variable spaces. 


\section{Variable Hardy and Hardy Lorentz spaces}

Now we introduce the variable Hardy and Hardy-Lorentz spaces and give their atomic decompositions. Denote by $S\left(\mathbb{R}^{d}\right)$ the space of all Schwartz functions and by $S^{\prime}\left(\mathbb{R}^{d}\right)$ the space of all tempered distributions. For $t \in(0, \infty)$ and $x \in \mathbb{R}^{d}$, let

$$
\psi_{t}(x):=t^{-d} \psi(x / t)
$$

Let $\psi \in S\left(\mathbb{R}^{d}\right)$ be a fixed Schwartz function with $\int_{\mathbb{R}^{d}} \psi d \lambda \neq 0$. The maximal function of a tempered distribution $f \in S^{\prime}\left(\mathbb{R}^{d}\right)$ is defined by

$$
f^{+}(x):=\sup _{0<t<\infty}\left|f * \psi_{t}(x)\right| \quad\left(x \in \mathbb{R}^{d}\right) .
$$

The variable Hardy and Hardy-Lorentz spaces $H_{p(\cdot)}\left(\mathbb{R}^{d}\right)$ and $H_{p(\cdot), q}\left(\mathbb{R}^{d}\right)$ are consisting of all tempered distributions $f \in S^{\prime}\left(\mathbb{R}^{d}\right)$ such that

$$
\|f\|_{H_{p(\cdot)}\left(\mathbb{R}^{d}\right)}:=\left\|f^{+}\right\|_{L_{p(\cdot)}\left(\mathbb{R}^{d}\right)}<\infty, \quad\|f\|_{H_{p(\cdot), q}\left(\mathbb{R}^{d}\right)}:=\left\|f^{+}\right\|_{L_{p(\cdot), q}\left(\mathbb{R}^{d}\right)}<\infty
$$

respectively. It is known that different functions $\psi$ give the same space with equivalent norms. Moreover, all $f \in H_{p(\cdot)}\left(\mathbb{R}^{d}\right)$ and $f \in H_{p(\cdot), q}\left(\mathbb{R}^{d}\right)$ are bounded distributions, i.e. $f * \phi \in L_{\infty}\left(\mathbb{R}^{d}\right)$ for all $\phi \in S\left(\mathbb{R}^{d}\right)$.

Theorem 5.1. If $p_{-}>1$, then

$$
H_{p(\cdot)}\left(\mathbb{R}^{d}\right) \sim L_{p(\cdot)}\left(\mathbb{R}^{d}\right), \quad H_{p(\cdot), q}\left(\mathbb{R}^{d}\right) \sim L_{p(\cdot), q}\left(\mathbb{R}^{d}\right) .
$$

For variable Hardy and Hardy-Lorentz spaces see the references Nakai and Sawano [21, 23], Yan at al. [35], Liu at al. [18, 19] and Jiao at al. [15]. If $p(\cdot)$ is a constant, then we get back the classical Hardy and Hardy-Lorentz spaces $H_{p}\left(\mathbb{R}^{d}\right)$ and $H_{p, q}\left(\mathbb{R}^{d}\right)$.

The atomic decomposition of variable Hardy spaces were studied in Nakai and Sawano [21, 23], Yan at al. [35], Liu at al. [18, 19] and Jiao at al. [15].

Definition 5.2. Let $p(\cdot) \in \mathcal{P}\left(\mathbb{R}^{d}\right)$ and fix an integer $d\left(1 / p_{-}-1\right)<s<\infty$. A measurable function $a$ is called a $p(\cdot)$-atom if there exists a ball $B \subset \mathbb{R}^{d}$ such that

(a) supp a $\subset$ B,

(b) $\|a\|_{L_{\infty}\left(\mathbb{R}^{d}\right)} \leq \frac{1}{\left\|\chi_{B}\right\|_{L_{p(\cdot)}\left(\mathbb{R}^{d}\right)}}$,

(c) $\int_{\mathbb{R}^{d}} a(x) x^{\alpha} d x=0$ for all multi-indices $\alpha$ with $|\alpha| \leq s$.

Theorem 5.3. Let $p(\cdot) \in C^{\log }\left(\mathbb{R}^{d}\right)$. A tempered distribution $f \in S^{\prime}\left(\mathbb{R}^{d}\right)$ is in $H_{p(\cdot)}\left(\mathbb{R}^{d}\right)$ if and only if there exist a sequence $\left\{a_{i}\right\}_{i \in \mathbb{N}}$ of $p(\cdot)$-atoms with support $\left\{B_{i}\right\}_{i \in \mathbb{N}}$ and a sequence $\left\{\lambda_{i}\right\}_{i \in \mathbb{N}}$ of positive numbers such that

$$
f=\sum_{i \in \mathbb{N}} \lambda_{i} a_{i} \quad \text { in } \quad S^{\prime}\left(\mathbb{R}^{d}\right) .
$$

Moreover,

$$
\|f\|_{H_{p(\cdot)}\left(\mathbb{R}^{d}\right)} \sim \inf \left\|\left(\sum_{i \in \mathbb{N}}\left(\frac{\lambda_{i} \chi_{B_{i}}}{\left\|\chi_{B_{i}}\right\|_{L_{p(\cdot)}\left(\mathbb{R}^{d}\right)}}\right)^{\underline{p}}\right)^{1 / \underline{p}}\right\|_{L_{p(\cdot)}\left(\mathbb{R}^{d}\right)},
$$


where the infimum is taken over all decompositions of $f$ as above.

Here we use the notation $p=\min \left\{p_{-}, 1\right\}$.

Theorem 5.4. Let $p(\cdot) \in C^{\log }\left(\mathbb{R}^{d}\right)$ and $0<q \leq \infty$. A tempered distribution $f \in S^{\prime}\left(\mathbb{R}^{d}\right)$ is in $H_{p(\cdot), q}\left(\mathbb{R}^{d}\right)$ if and only if there exist a sequence $\left\{a_{i, j}\right\}_{i \in \mathbb{Z}, j \in \mathbb{N}}$ of $p(\cdot)$-atoms with support $\left\{B_{i, j}\right\}_{i \in \mathbb{Z}, j \in \mathbb{N}}$ such that

$$
f=\sum_{i \in \mathbb{Z}} \sum_{j \in \mathbb{N}} \lambda_{i, j} a_{i, j} \quad \text { in } \quad S^{\prime}\left(\mathbb{R}^{d}\right),
$$

where $\sum_{j \in \mathbb{N}} \chi_{B_{i, j}}(x) \leq A$ for all $x \in \mathbb{R}^{d}$ and $i \in \mathbb{Z}$ and $\lambda_{i, j}:=C 2^{i}\left\|\chi_{B_{i, j}}\right\|_{L_{p(\cdot)}\left(\mathbb{R}^{d}\right)}$ $(i \in \mathbb{Z}, j \in \mathbb{N}$ ) with $A$ and $C$ being positive constants. Moreover,

$$
\|f\|_{H_{p(\cdot), q}\left(\mathbb{R}^{d}\right)} \sim \inf \left(\sum_{i \in \mathbb{Z}}\left\|\left(\sum_{j \in \mathbb{N}}\left(\frac{\lambda_{i, j} \chi_{B_{i, j}}}{\left\|\chi_{B_{i, j}}\right\|_{L_{p(\cdot)}\left(\mathbb{R}^{d}\right)}}\right)^{\underline{p}}\right)^{1 / \underline{p}}\right\|_{L_{p(\cdot)}\left(\mathbb{R}^{d}\right)}^{q}\right)^{1 / q},
$$

where the infimum is taken over all decompositions of $f$ as above.

\section{Summability in $H_{p(\cdot)}\left(\mathbb{R}^{d}\right)$ and $H_{p(\cdot), q}\left(\mathbb{R}^{d}\right)$}

In this section, we will investigate the boundedness of some operators from $H_{p(\cdot)}\left(\mathbb{R}^{d}\right)$ to $L_{p(\cdot)}\left(\mathbb{R}^{d}\right)$ and from $H_{p(\cdot), q}\left(\mathbb{R}^{d}\right)$ to $L_{p(\cdot), q}\left(\mathbb{R}^{d}\right)$ (see Weisz [30]).

Theorem 6.1. Let $p(\cdot) \in C^{\log }\left(\mathbb{R}^{d}\right), 0<q<\infty, \gamma>1$ and $p_{-}>1 / \gamma$. For each $t>0$ let $K_{t} \in L_{1}\left(\mathbb{R}^{d}\right)$ and $V_{t} f=f * K_{t}$. Suppose that

$$
\left|V_{*} a(x)\right| \leq C\left\|\chi_{B}\right\|_{L_{p(\cdot)}^{-1}\left(\mathbb{R}^{d}\right)}^{-}\left|M \chi_{B}(x)\right|^{\gamma} \quad(x \notin 2 B)
$$

for all $p(\cdot)$-atoms a with support $B$. If $V_{*}$ is bounded from $L_{\infty}\left(\mathbb{R}^{d}\right)$ to $L_{\infty}\left(\mathbb{R}^{d}\right)$, then

$$
\left\|V_{*} f\right\|_{L_{p(\cdot)}\left(\mathbb{R}^{d}\right)} \leq C\|f\|_{H_{p(\cdot)}\left(\mathbb{R}^{d}\right)} \quad\left(f \in H_{p(\cdot)}\left(\mathbb{R}^{d}\right)\right)
$$

and

$$
\left\|V_{*} f\right\|_{L_{p(\cdot), q}\left(\mathbb{R}^{d}\right)} \lesssim\|f\|_{H_{p(\cdot), q}\left(\mathbb{R}^{d}\right)} \quad\left(f \in H_{p(\cdot), q}\left(\mathbb{R}^{d}\right)\right) .
$$

Using Theorem 4.1, we can show easily that (6.1) implies (3.4). Now let us apply the result for the summability of Fourier transforms. It is known that $\sigma_{T}^{\theta}$ is bounded from $L_{1}\left(\mathbb{R}^{d}\right)$ to $L_{1}\left(\mathbb{R}^{d}\right)$ for all $T>0$ and $\sigma_{*}^{\theta}$ is bounded from $L_{\infty}\left(\mathbb{R}^{d}\right)$ to $L_{\infty}\left(\mathbb{R}^{d}\right)$ (see e.g. Weisz [31]). The next theorem shows that the additional condition (6.2) implies (6.1) (see Weisz [30]).

Theorem 6.2. Let (2.3) be satisfied. Assume that $\widehat{\theta_{0}}$ is $(N+1)$-times differentiable for some $N \in \mathbb{N}$ and there exists $d+N<\beta \leq d+N+1$ such that

$$
\left|\partial_{1}^{i_{1}} \ldots \partial_{d}^{i_{d}} \widehat{\theta_{0}}(x)\right| \leq C|x|^{-\beta} \quad(x \neq 0)
$$

whenever $i_{1}+\ldots+i_{d}=N$ or $i_{1}+\ldots+i_{d}=N+1$. If $p(\cdot) \in C^{\log }\left(\mathbb{R}^{d}\right)$, then

$$
\left|\sigma_{*}^{\theta} a(x)\right| \leq C\left\|\chi_{B}\right\|_{L_{p(\cdot)}\left(\mathbb{R}^{d}\right)}^{-1}\left|M \chi_{B}(x)\right|^{\beta / d}
$$


for all $p(\cdot)$-atoms $a$ and all $x \notin 2 B$, where the ball $B$ is the support of the atom.

The following result follows from Theorems 6.1 and 6.2. Note that $\beta>d$.

Corollary 6.3. Let (2.3) and (6.2) be satisfied. If $p(\cdot) \in C^{\log }\left(\mathbb{R}^{d}\right), 0<q<\infty$ and $p_{-}>d / \beta$, then

$$
\left\|\sigma_{*}^{\theta} f\right\|_{L_{p(\cdot)}\left(\mathbb{R}^{d}\right)} \lesssim\|f\|_{H_{p(\cdot)}\left(\mathbb{R}^{d}\right)} \quad\left(f \in H_{p(\cdot)}\left(\mathbb{R}^{d}\right)\right)
$$

and

$$
\left\|\sigma_{*}^{\theta} f\right\|_{L_{p(\cdot), q}\left(\mathbb{R}^{d}\right)} \lesssim\|f\|_{H_{p(\cdot), q}\left(\mathbb{R}^{d}\right)} \quad\left(f \in H_{p(\cdot), q}\left(\mathbb{R}^{d}\right)\right) .
$$

Note that if $p(\cdot)$ is a constant, then we get back the classical result (see Weisz $[31,32]$ as well as Corollary 3.6). Using Corollary 6.3 and a usual density argument, we obtain the next convergence results (see Weisz [30]).

Corollary 6.4. Suppose that (2.3) and (6.2) are satisfied, $p(\cdot) \in C^{\log }\left(\mathbb{R}^{d}\right), 0<q<\infty$ and $p_{-}>d / \beta$. If $f \in H_{p(\cdot)}\left(\mathbb{R}^{d}\right)$ (resp. $f \in H_{p(\cdot), q}\left(\mathbb{R}^{d}\right)$ ), then $\sigma_{T}^{\theta} f$ converges almost everywhere as well as in the $L_{p(\cdot)}\left(\mathbb{R}^{d}\right)$-norm (resp. in the $L_{p(\cdot), q}\left(\mathbb{R}^{d}\right)$-norm) as $T \rightarrow \infty$.

Corollary 6.5. Suppose that (2.3) and (6.2) are satisfied, $p(\cdot) \in C^{\log \left(\mathbb{R}^{d}\right) \text { and } 1 \leq q<}$ $\infty$. If $p_{-}>1$ and $f \in L_{p(\cdot)}\left(\mathbb{R}^{d}\right)$ (resp. $f \in L_{p(\cdot), q}\left(\mathbb{R}^{d}\right)$ ), then

$$
\lim _{T \rightarrow \infty} \sigma_{T}^{\theta} f(x)=f(x) \quad \text { for a.e. } x \in \mathbb{R}^{d}
$$

as well as in the $L_{p(\cdot)}\left(\mathbb{R}^{d}\right)$-norm (resp. in the $L_{p(\cdot), q}\left(\mathbb{R}^{d}\right)$-norm). The almost everywhere convergence holds also if $f \in L_{p(\cdot)}\left(\mathbb{R}^{d}\right)$ with $p_{-} \geq 1$.

\section{Some summability methods}

As special cases, we consider some summability methods.

\subsection{Riesz summation}

The function

$$
\theta_{0}(t)=\left\{\begin{array}{ll}
\left(1-|t|^{\gamma}\right)^{\alpha}, & \text { if }|t|>1 \\
0, & \text { if }|t| \leq 1
\end{array} \quad\left(t \in \mathbb{R}^{d}\right)\right.
$$

defines the Riesz summation if $0<\alpha<\infty$ and $\gamma$ is a positive integer. It is called Bochner-Riesz summation if $\gamma=2$ and Fejr summation if $\alpha=\gamma=1$. The following result follows from Corollaries 6.3-6.5.

Corollary 7.1. If $p(\cdot) \in C^{\log }\left(\mathbb{R}^{d}\right), 0<q<\infty$ and

$$
\alpha>\frac{d-1}{2}, \quad \frac{d}{d / 2+\alpha+1 / 2}<p_{-}<\infty,
$$

then

and

$$
\left\|\sigma_{*}^{\theta} f\right\|_{L_{p(\cdot)}\left(\mathbb{R}^{d}\right)} \lesssim\|f\|_{H_{p(\cdot)}\left(\mathbb{R}^{d}\right)} \quad\left(f \in H_{p(\cdot)}\left(\mathbb{R}^{d}\right)\right)
$$

$$
\left\|\sigma_{*}^{\theta} f\right\|_{L_{p(\cdot), q}\left(\mathbb{R}^{d}\right)} \lesssim\|f\|_{H_{p(\cdot), q}\left(\mathbb{R}^{d}\right)} \quad\left(f \in H_{p(\cdot), q}\left(\mathbb{R}^{d}\right)\right) .
$$

Moreover, the corresponding Corollaries 6.4-6.5 hold as well. 


\subsection{Weierstrass summation}

The Weierstrass summation is defined by

$$
\theta_{0}(t)=e^{-|t|^{2} / 2} \quad \text { or } \quad \theta_{0}(t)=e^{-|t|} \quad\left(t \in \mathbb{R}^{d}\right) .
$$

Corollary 7.2. If $p(\cdot) \in C^{\log }\left(\mathbb{R}^{d}\right)$ and $0<q<\infty$, then

$$
\left\|\sigma_{*}^{\theta} f\right\|_{L_{p(\cdot)}\left(\mathbb{R}^{d}\right)} \lesssim\|f\|_{H_{p(\cdot)}\left(\mathbb{R}^{d}\right)} \quad\left(f \in H_{p(\cdot)}\left(\mathbb{R}^{d}\right)\right)
$$

and

$$
\left\|\sigma_{*}^{\theta} f\right\|_{L_{p(\cdot), q}\left(\mathbb{R}^{d}\right)} \lesssim\|f\|_{H_{p(\cdot), q}\left(\mathbb{R}^{d}\right)} \quad\left(f \in H_{p(\cdot), q}\left(\mathbb{R}^{d}\right)\right) .
$$

Moreover, the corresponding Corollaries 6.4-6.5 hold as well.

\subsection{Picard-Bessel summation}

Corollary 7.2 holds for the summability method defined by

$$
\theta_{0}(t)=\left(1+|t|^{2}\right)^{-(d+1) / 2} \quad\left(t \in \mathbb{R}^{d}\right) .
$$

\section{References}

[1] Bownik, M., Boundedness of operators on Hardy spaces via atomic decompositions, Proc. Amer. Math. Soc., 133(2005), 3535-3542.

[2] Butzer, P.L., Nessel, R.J., Fourier Analysis and Approximation, Birkhäuser Verlag, Basel, 1971.

[3] Carleson, L., On convergence and growth of partial sums of Fourier series, Acta Math., 116(1966), 135-157.

[4] Cruz-Uribe, D., Fiorenza, A., Martell, J., Pérez, C., The boundedness of classical operators on variable $L^{p}$ spaces, Ann. Acad. Sci. Fenn. Math., 31(2006), 239-264.

[5] Cruz-Uribe, D.V., Fiorenza, A., Variable Lebesgue Spaces. Foundations and Harmonic Analysis, New York, Birkhäuser/Springer, 2013.

[6] Diening, L., Harjulehto, P., Hästö, P., Ružička, M., Lebesgue and Sobolev Spaces with Variable Exponents, Berlin, Springer, 2011.

[7] Feichtinger, H.G., Weisz, F., Wiener amalgams and pointwise summability of Fourier transforms and Fourier series, Math. Proc. Cambridge Philos. Soc., 140(2006), 509-536.

[8] Gát, G., Pointwise convergence of cone-like restricted two-dimensional $(C, 1)$ means of trigonometric Fourier series, J. Approx. Theory., 149(2007), 74-102.

[9] Gát, G., Goginava, U., Nagy, K., On the Marcinkiewicz-Fejér means of double Fourier series with respect to Walsh-Kaczmarz system, Studia Sci. Math. Hungar., 46(2009), 399-421.

[10] Goginava, U., Marcinkiewicz-Fejér means of d-dimensional Walsh-Fourier series, J. Math. Anal. Appl., 307(2005), 206-218.

[11] Goginava, U., Almost everywhere convergence of $(C, a)$-means of cubical partial sums of d-dimensional Walsh-Fourier series, J. Approx. Theory, 141(2006), 8-28.

[12] Grafakos, L., Classical and Modern Fourier Analysis, Pearson Education, New Jersey, 2004.

[13] Hunt, R.A., On the convergence of Fourier series, in: Orthogonal Expansions and their Continuous Analogues, Proc. Conf. Edwardsville, Ill., 1967, pages 235-255, Illinois Univ. Press Carbondale, 1968. 
[14] Jiao, Y., Zhou, D., Weisz, F., Wu, L., Variable martingale Hardy spaces and their applications in Fourier analysis, (preprint).

[15] Jiao, Y., Zuo, Y., Zhou, D., Wu, L., Variable Hardy-Lorentz spaces $H^{p(\cdot), q}\left(\mathbb{R}^{n}\right)$, Math. Nachr., (to appear).

[16] Kempka, H., Vybíral, J., Lorentz spaces with variable exponents, Math. Nachr., 287(2014), 938-954.

[17] Latter, R.H., A characterization of $H^{p}\left(\mathbf{R}^{n}\right)$ in terms of atoms, Studia Math., 62(1978), $92-101$.

[18] Liu, J., Weisz, F., Yang, D., Yuan, W., Littlewood-Paley and finite atomic characterizations of anisotropic variable Hardy-Lorentz spaces and their applications, J. Fourier Anal. Appl., (to appear).

[19] Liu, J., Weisz, F., Yang, D., Yuan, W., Variable anisotropic Hardy spaces and their applications, Taiwanese J. Math., (to appear).

[20] Lu, S., Four Lectures on Real $H^{p}$ Spaces, World Scientific, Singapore, 1995.

[21] Nakai, E., Sawano, Y., Hardy spaces with variable exponents and generalized Campanato spaces, J. Funct. Anal., 262(2012), no. 9, 3665-3748.

[22] Persson, L.E., Tephnadze, G., Wall, P., Maximal operators of Vilenkin-Nörlund means, J. Fourier Anal. Appl., 21(1015), no. 1, 76-94.

[23] Sawano, Y., Atomic decompositions of Hardy spaces with variable exponents and its application to bounded linear operators, Integral Equations Operator Theory, 77(2013), 123-148.

[24] Simon, P., $(C, \alpha)$ summability of Walsh-Kaczmarz-Fourier series, J. Approx. Theory, 127(2004), 39-60.

[25] Simon, P., On a theorem of Feichtinger and Weisz, Ann. Univ. Sci. Budap. Rolando Eötvös, Sect. Comput., 39(2013), 391-403.

[26] Stein, E.M., Harmonic Analysis: Real-variable Methods, Orthogonality and Oscillatory Integrals, Princeton Univ. Press, Princeton, N.J., 1993.

[27] Stein, E.M., Taibleson, M.H., Weiss, G., Weak type estimates for maximal operators on certain $H^{p}$ classes, Rend. Circ. Mat. Palermo, Suppl., 1(1981), 81-97.

[28] Stein, E.M., Weiss, G., Introduction to Fourier Analysis on Euclidean Spaces, Princeton Univ. Press, Princeton, N.J., 1971.

[29] Trigub, R.M., Belinsky, E.S., Fourier Analysis and Approximation of Functions, Kluwer Academic Publishers, Dordrecht, Boston, London, 2004.

[30] Weisz, F., Summability of Fourier transforms in variable Hardy and Hardy-Lorentz spaces, (preprint).

[31] Weisz, F., Summability of Multi-dimensional Fourier Series and Hardy Spaces, Mathematics and Its Applications. Kluwer Academic Publishers, Dordrecht, Boston, London, 2002.

[32] Weisz, F., Summability of multi-dimensional trigonometric Fourier series, Surv. Approx. Theory, 7(2012), 1-179.

[33] Weisz, F., Convergence and Summability of Fourier Transforms and Hardy Spaces, Applied and Numerical Harmonic Analysis, Springer, Birkhäuser, Basel, 2017.

[34] Wilson, J.M., On the atomic decomposition for Hardy spaces, Pac. J. Math., 116(1985), 201-207. 
[35] Yan, X., Yang, D., Yuan, W., Zhuo, C., Variable weak Hardy spaces and their applications, J. Funct. Anal., 271(2016), 2822-2887.

Ferenc Weisz

Department of Numerical Analysis, Eötvös L. University

H-1117 Budapest, Pázmány P. sétány 1/C., Hungary

e-mail: weisz@inf.elte.hu 